\title{
Synchronization of Chaotic Systems: A Generic Nonlinear Integrated Observer-Based Approach
}

\author{
Muhammad Majid Hussain $\mathbb{D}^{1},{ }^{1}$ Muhammad Siddique $\mathbb{D}^{2},{ }^{2}$ Ziyad M. Almohaimeed $\mathbb{D},{ }^{3}$ \\ Romaisa Shamshad, ${ }^{2}$ Rizwan Akram $\mathbb{D}^{3},{ }^{3}$ and Naeem Aslam (iD) \\ ${ }^{1}$ Department of Electrical and Electronic Engineering, University of South Wales, Pontypirdd CF37 1DL, UK \\ ${ }^{2}$ Department of Electrical Engineering \& Computer Science, NFC Institute of Engineering and Technology (NFC-IET), \\ Multan 66000, Pakistan \\ ${ }^{3}$ Department of Electrical Engineering, College of Engineering, Qassim University, P.O.Box 6677, Qassim 51452, Saudi Arabia
}

Correspondence should be addressed to Muhammad Siddique; msiddique@nfciet.edu.pk and Rizwan Akram; rizwanakram75@qec.edu.sa

Received 3 October 2021; Revised 3 November 2021; Accepted 6 November 2021; Published 2 December 2021

Academic Editor: Jawad Ahmad

Copyright ( 92021 Muhammad Majid Hussain et al. This is an open access article distributed under the Creative Commons Attribution License, which permits unrestricted use, distribution, and reproduction in any medium, provided the original work is properly cited.

The purpose of this research is to study the synchronization of two integrated nonlinear systems with time delay and disturbances. A nonlinear system is a system in which the difference in output is not relative to the difference in input. A new control methodology for synchronization of the two chaotic systems master and slave is recognized by means of the unique integrated chaotic synchronous observer and the integrated chaotic adaptive synchronous observer. The instantaneous approximation states of the master and slave systems are accomplished by means of methods for suggesting observers for every one of the master and slave systems and by the production of error signals between these approximated states. This approximated synchronization error signal and state approximation errors meet at the origin by means of methods involving a particular observer-based feedback control signal to ensure synchronization and state approximation. Using Lyapunov stability theory, adaptive and nonadaptive laws for control systems, and nonlinear properties, the intermingling conditions for state approximation errors and approximated synchronization errors are established as nonlinear matrix inequalities. A solution to the resulting inequality constraints using a two-step linear matrix inequality (LMI)-based approach is introduced, giving essential and adequate conditions to extract values from the controller gain and observer gain matrices. Simulation of the suggested synchronization procedure for FitzHugh-Nagumo neuronal systems is demonstrated to expand the viability of the suggested observer-based control techniques.

\section{Introduction}

In nature, most real systems are nonlinear. To better understand the performance of distinctive nonlinear systems, it is significant and interesting to study the synchronization between two systems. Synchronization, perceived as a procedure that normally happens, has a notable effect in different areas of science, design, and engineering, even in public activities. Nonlinear system synchronization is an interesting field amid specialisations in various trains of thought because of its various uses relating to design and innovation. Researchers stepped into the universe of nonlinear systems in 1988, and various papers were published on the subject $[1,2]$. Nonlinear systems do not obey the principle of superposition and their output is not directly proportional to their input. Pecora and Carroll were responsible for the earliest effective work on the subject, introducing an experiment for synchronization of nonlinear systems under various initial conditions. Pecora and Carroll published a seminal paper [3] in the field of nonlinear synchronization. In this study, they described that certain nonlinear chaotic systems can be made to synchronize by linking them with common signals. The criterion for this is the sign of the sub-Lyapunov exponents. We apply these ideas to a real set of synchronizing chaotic circuits. Subsequently, scientists have developed numerous nonlinear 
synchronization strategies. The process of synchronization is where the determined system (slave system) comes to be in parallel with the master system (driving system), meaning that the synchronized system moves in a specific way, following the direction of a synchronizing system $[4,5]$. The background of this article outlines that many different methodologies have been used, including the Runge-Kutta model-based nonlinear observer [6], linear feedback control (LFC) [7], and delay-range dependent methodologies [8, 9]. Adaptive schemes using fuzzy disturbance observers [10], robust adaptive methodology $[11,12]$, reduced-order and full-order output-related observers [13], synchronization with Huygens' coupling [14], adaptive generalized projective synchronization (GPS) [15], stepwise sliding mode observer techniques [16], evolutionary algorithms [17], backstepping techniques [18], and nonlinear synchronism of undefined inputs, as well as Takagi-Sugeno fuzzy [19], have all been implemented for the coordination of chaos systems. All of these defined methods of synchronization of nonlinear modules show their robustness to different technologies such as neural networks [20], biological systems [21], secure communication [22, 23], robotics [24], optics and lasers [25], information science [26], and chemical reaction [27]. Observer-based synchronization methods are progressively pertinent to the condition, where the master and slave situations are unknown [28]. Research specialists are consistently investigating such methods with various kinds of observers for different applications, for example, synchronous chaos in coupled systems [29], comprehensive projective synchronization procedures dependent on state approximation of hyperchaotic modules without computing Lyapunov proponents, and nonlinear-based protective communication, using decreased-order and stepwise sliding state observers. Nevertheless, previous statements of observer-dependent synchronization methods do not explain the integrated chaotic synchronized (ICS) observer and integrated chaotic adaptive synchronized (ICAS) observerdependent control strategies shown in this article. The primary disadvantage of the strategies previously mentioned, as opposed to the ICS and ICAS observer-dependent control techniques, is their appropriateness for the lower degree of synchronization of the two nonlinear modules with inaccessible state vectors. An error concurrent observer-dependent synchronization method was suggested in a recent work [30]. However, the technique is only used in nonlinear modules for which the general error module is adaptable to a direct composition of several error parameters. This is widely used in applications to secure communications. The numerous forms of chaotic synchronization include synchronization of Lur'e master and slave system. The work behind this synchronization of the chaotic Lur'e system was controlled in different ways. The absolute stability theory and different circumstances have been established. The objective of this research paper is to synchronize the unbalanced master pendulum system and slave system using a robust feedback technique and the LMIbased method for the synchronization of the chaotic dynamical pendulum system and output feedback controller technique. The main contribution and the objectives of the paper are (i) development of robust adaptive feedback control for delay containing chaotic systems, (ii) the mitigation of the effect of the disturbances using novel integrated adaptive observers, and (iii) a sufficient condition for the existence of observer and controller gains for the synchronization of chaotic systems. The closed-loop error is minimized after very little time and the system becomes stable, so the disturbance input effect reduces. To validate our research results, we have considered the example of the phase-locked loop system.

\section{System Description}

Synchronization of nonlinear systems is a subject matter. It means that synchronization of the dynamics of those systems occur, containing nonlinearities in their dynamics. Mathematical representations of nonlinear systems, which will be synchronized, contain both types of nonlinearities mentioned. Following this discussion, it is necessary to consider the generalized model of nonlinear master and slave chaotic (nonlinear) system equations (1) and (2), defined by state space representation when disturbance and adaptation are zero. $d m=0 ; d s=0$.

$$
\begin{aligned}
\dot{x}_{m}(t)= & A x_{m}(t)+A_{d} x_{m}(t-\tau)+f\left(x_{m}(t)\right)+f_{d}\left(x_{m}(t-\tau)\right)+B g\left(x_{m}(t)\right) \theta_{m} \\
& +B g_{d}\left(x_{m}(t-\tau)\right) \theta_{m, d}+d_{m}, y_{m}(t)=C x_{m}(t), \\
\dot{x}_{s}(t)= & A x_{s}(t)+A_{d} x_{s}(t-\tau)+f\left(x_{s}(t)\right)+f_{d}\left(x_{s}(t-\tau)\right)+B g\left(x_{s}(t)\right) \theta_{s} \\
& +B g_{d}\left(x_{s}(t-\tau)\right) \theta_{s, d}+d_{s}+B u(t), \\
y_{s}(t)= & C x_{s}(t),
\end{aligned}
$$

where $x m(t) \in \mathrm{Rn}$ and $x s(t) \in \mathrm{Rn}$ are the state vectors for the master and slave systems, respectively. Similarly, $y m(t) \in R m$ and $y s(\mathrm{t}) \in \mathrm{Rm}$ are the output vectors. $A, \in R^{n \times n}, \mathrm{~B}, \in \mathrm{R}^{n \times 1}$, and $\mathrm{C}, \in R, m \times n$ are real constant matrices. The vector functions $f(\mathrm{x}(\mathrm{t})) \in \mathrm{Rn}$ and $g(x(t)) \in \mathrm{R}^{\mathrm{R} \times \mathrm{p}}$ are the nonlinear functions $(\tau-\mathrm{t})$. Nonlinear function time delay, $q m(t) \in \mathrm{Rp}$ and $q s(t) \in \mathrm{Rp}$ are the unknown parameters in the dynamics of the chaotic oscillators, and $\mathrm{u}(\mathrm{t}) \in \mathrm{Rl}$ is the control input. 


\section{Integrated Adaptive Observer- Dependent Synchronization}

If unknown parameters such as $\theta_{m}, \theta_{s}, \theta_{m, d}, \theta_{s, d} \in R^{p}$ are present in the master $(\mathrm{M})$ and slave $(\mathrm{S})$ nonlinear systems, then the control law will not be applicable for the synchronization of both systems. In such cases, we will use adaptation laws with a choice of control law:

$$
\begin{aligned}
u(t)= & \psi\left(\widehat{x}_{m}(t), \hat{x}_{s}(t)\right) \Psi\left(\widehat{x}_{m}(t), \widehat{x}_{s}(t)\right)=F\left(\widehat{x}_{m}(t)-\widehat{x}_{s}(t)\right)+g\left(\widehat{x}_{m}(t)\right) \widehat{\theta}_{m}(t)-g\left(\hat{x}_{s}(t)\right) \widehat{\theta}_{s}(t) \\
& +g_{d}\left(\widehat{x}_{m}(t-\tau)\right) \widehat{\theta}_{m, d}(t-\tau)-g_{d}\left(\widehat{x}_{s}(t-\tau)\right) \widehat{\theta}_{s, d}(t-\tau),
\end{aligned}
$$

where $\quad \hat{\theta}_{m}(t) \in R^{p}, \quad \hat{\theta}_{s}(t) \in R^{p}, \quad \hat{\theta}_{m, d}(t-\tau) \in R^{p}$, $\hat{\theta}_{s, d}(t-\tau) \in R^{p}$, and $\theta_{m}, \theta_{s}, \theta_{m, d}$, and $\theta_{s, d}, \in R^{p}$ are the unknown parameter estimates. For the estimation of both system states under unspecified parameters, coupled adaptive observers are the best to use:

$$
\begin{aligned}
\dot{\hat{x}}_{m}(t)= & A \widehat{x}_{m}(t)+A_{d} \widehat{x}_{m}(t-\tau)+f\left(\widehat{x}_{m}(t)\right)+f_{d}\left(\widehat{x}_{m}(t-\tau)\right)+B g\left(\widehat{x}_{m}(t)\right) \widehat{\theta}_{m}(t)+B g_{d}\left(\widehat{x}_{m}(t-\tau)\right) \widehat{\theta}_{m, d}(t-\tau) \\
& +L_{m}\left(y_{m}(t)-\widehat{y}_{m}(t)\right)-\frac{1}{2} B F\left(\widehat{x}_{m}(t)-\widehat{x}_{s}(t)\right), \\
\dot{\hat{x}}_{s}(t)= & A \widehat{x}_{s}(t)+A_{d} \widehat{x}_{s}(t-\tau)+f\left(\widehat{x}_{s}(t)\right)+f_{d}\left(\widehat{x}_{s}(t-\tau)\right)+B g\left(\widehat{x}_{s}(t)\right) \hat{\theta}_{s}(t)+B g_{d}\left(\hat{x}_{s}(t-\tau)\right) \widehat{\theta}_{s, d}(t-\tau) \\
& +L_{s}\left(y_{s}(t)-\widehat{y}_{s}(t)\right)-\frac{1}{2} B F\left(\widehat{x}_{m}(t)-\widehat{x}_{s}(t)\right)+B u(t),
\end{aligned}
$$

where $L_{m} \in R^{n \times m}$ and $L_{s} \in R^{n \times m}$ are the observer gain matrices. With the help of equation (3), we can manage the model structure of the slave (S) observer given as

$$
\begin{aligned}
\dot{\hat{x}}_{s}(t)= & A \widehat{x}_{s}(t)+A_{d} \widehat{x}_{s}(t-\tau)+f\left(\widehat{x}_{s}(t)\right)+f_{d}\left(\widehat{x}_{s}(t-\tau)\right)+B g\left(\widehat{x}_{s}(t)\right) \hat{\theta}_{s}(t) \\
& +B g_{d}\left(\widehat{x}_{s}(t)\right) \widehat{\theta}_{s, d}(t-\tau)+L_{s}\left(y_{s}(t)-\widehat{y}_{s}(t)\right)+\frac{1}{2} B F\left(\widehat{x}_{m}(t)-\widehat{x}_{s}(t)\right)+B u_{g}(t), \\
u_{g}(t)= & g\left(\widehat{x}_{m}(t)\right) \hat{\theta}_{m}(t)-g\left(\hat{x}_{s}(t)\right) \hat{\theta}_{s}(t)+g_{d}\left(\widehat{x}_{m}(t-\tau)\right) \hat{\theta}_{m, d}(t-\tau)-g_{d}\left(\hat{x}_{s}(t-\tau)\right) \widehat{\theta}_{s, d}(t-\tau),
\end{aligned}
$$

where $u(t)$ is a nonlinear element. Also, in addition, we define

$$
\begin{aligned}
e_{m}(t) & =x_{m}(t)-\widehat{x}_{m}(t), \\
e_{s}(t) & =x_{s}(t)-\widehat{x}_{s}(t), \\
e_{0}(t) & =\widehat{x}_{m}(t)-\widehat{x}_{s}(t), \\
\psi(t) & =f(x(t))-f(\widehat{x}(t)), \\
\psi_{d}(t, \tau) & =f_{d}(x(t-\tau))-f_{d}(\widehat{x}(t-\tau)), \\
\psi_{g}(t) & =B g\left(x_{m}(t)\right) \theta_{m}-B g\left(\widehat{x}_{m}(t)\right) \widehat{\theta}_{m}(t), \\
\psi_{(g, d)}(t, \tau) & =B g_{d}(x(t-\tau)) \theta_{d}-B g_{d}(\widehat{x}(t-\tau)) \widehat{\theta}_{d}(t-\tau) .
\end{aligned}
$$

\section{Synchronization Feedback Control}

The (M) and (S) chaotic frameworks can be made clear through the use of their particular observers. These observers for the (M) and (S) frameworks produce evaluations of the conditions of the particular framework. The two approximates of the $(\mathrm{M})$ and $(\mathrm{S})$ frameworks are authorized to make up for a similar performance. This is finished by applying the recommended control holding the approximated conditions of the drive and reaction frameworks. The suggested control coordinates related approximated conditions of the two frameworks with the end goal that approximated the synchronization error, for example, $e_{s}(t)=x_{s}(t)-\widehat{x}_{s}(t)$ deals to zero. When it occurs, the conditions of the (M) and (S) observers remain synchronized. Subsequently, (M) and (S) observers are called synchronous observers. Presently, 
taking the derivative of both sides of the master, slave, and output error equation, we acquire

$$
\begin{aligned}
& \dot{e}_{m}(t)=A e_{m}(t)+A_{d} e_{m}(t-\tau)+\psi_{m}(t)+\psi_{d m}(t)+\psi_{g m}(t)+\psi_{(g, d) m}(t)-L_{m} C e_{m}(t)+d_{m}+\frac{1}{2} B F e_{o}(t), \\
& \dot{e}_{s}(t)=A e_{s}(t)+A_{d} e_{s}(t-\tau)+\psi_{s}(t)+\psi_{d s}(t)+\psi_{g s}(t)+\psi_{(g, d) s}(t)-L_{s} C e_{s}(t)-\frac{1}{2} B F e_{o}(t)+d_{s}-B u_{g}(t),
\end{aligned}
$$

where $u_{g}(t)$ is the nonlinear part of the proposed control law, i.e.,

$$
\begin{aligned}
& u_{g}(t)=g\left(\widehat{x}_{m}(t)\right) \widehat{\theta}_{m}(t)-g\left(\widehat{x}_{s}(t)\right) \widehat{\theta}_{s}(t)+g_{d}\left(\widehat{x}_{m}(t-\tau)\right) \widehat{\theta}_{m, d}(t-\tau)-g_{d}\left(\widehat{x}_{s}(t-\tau)\right) \widehat{\theta}_{s, d}(t-\tau), \\
& \dot{e}_{o}(t)=A e_{o}(t)+A_{d} e_{o}(t-\tau)+\psi_{o}(t)+\psi_{d o}(t)+L_{m} C e_{m}(t)-L_{s} C e_{s}(t)-B F e_{o}(t) .
\end{aligned}
$$

The assumption considers $B^{T} P_{m} C^{\perp}=0, B^{T} P_{s} C^{\perp}=0$, and $B^{T} P_{o} C^{\perp}=0$, where $C^{\perp}$ stands for the orthogonal projection on the null of $C$. If the above assumption holds, solving $\quad B^{T} P_{m}-R_{m} C=0, \quad B^{T} P_{s}-R_{s} C=0, \quad$ and $B^{T} P_{o}-R_{o} C=0$ matrices, $R_{m}, R_{s}$, and $R_{o}$ can be delegated. Adaptive controller design is provided using ICAS observers.

\section{Theorem}

The given observer and controller are then able to gain matrices $F \in R^{l \times n}, L_{m} \in R^{n \times m}$, and $L_{s} \in R^{n \times m}$ an appropriate state for synchronization of the (M) and (S) systems (1) and (2) with undefined dynamics $\theta_{m} \in R^{p}, \theta_{s} \in R^{p}, \theta_{m, d} \in R^{p}$, and $\theta_{s, d} \in R^{p}$ which concern with the assumption, applying the control law and ICAS observers (4)-(5), together with the law of adaptation:

$$
\begin{aligned}
\dot{\hat{\theta}}_{m}(t) & =-\Theta_{m} g^{T}\left(\hat{x}_{m}(t)\right) R_{m}\left(y_{m}(t)-C \hat{x}_{m}(t)\right), \quad \Theta_{m}>0, \\
\dot{\hat{\theta}}_{s}(t) & =-\Theta_{s} g^{T}\left(\hat{x}_{s}(t)\right) R_{s}\left(y_{s}(t)-C \hat{x}_{s}(t)\right), \quad \Theta_{s}>0, \\
\dot{\hat{\theta}}_{m, d}(t-\tau) & \left.\left.=-\Theta_{m, d} g_{d}^{T}\left(\hat{x}_{m}(t-\tau)\right) R_{m} y_{m}(t-\tau)\right)-C \widehat{x}_{m}(t-\tau)\right), \quad \Theta_{m, d}>0, \\
\dot{\hat{\theta}}_{s, d}(t-\tau) & \left.\left.=-\Theta_{s, d} g_{d}^{T}\left(\hat{x}_{s}(t-\tau)\right) R_{s} y_{s}(t-\tau)\right)-C \hat{x}_{s}(t-\tau)\right), \quad \Theta_{s, d}>0 .
\end{aligned}
$$

These are the adaptation rate $\dot{\hat{\theta}}_{m}, \dot{\hat{\theta}}_{s}, \dot{\hat{\theta}}_{m, d}$, and $\dot{\hat{\theta}}_{s, d}$ of proper quantities, with $P_{m}, P_{s}$, and $P_{o}$ positive-definite matrices. The scalars are $\alpha_{1}>0, \quad \alpha_{2}>0$, $\alpha_{3}>0, \alpha_{4}>0, \alpha_{5}>0, \alpha_{6}>0, \beta_{1}>0, \beta_{12}>0, \beta_{3}>0$, and $\beta_{4}>0$ so that the inequality matrix is satisfied:

$$
\left[\begin{array}{lll}
\Phi_{11_{9 \times 9}} & \Phi_{12_{9 \times 9}} & \Phi_{13_{9 \times 7}} \\
\Phi_{21_{9 \times 9}} & \Phi_{22_{9 \times 9}} & \Phi_{23_{9 \times 7}} \\
\Phi_{31_{9 \times 9}} & \Phi_{32_{9 \times 9}} & \Phi_{33_{7 \times 7}}
\end{array}\right]_{25 * 25}
$$

where

$$
\begin{aligned}
& \zeta_{11, m}=A^{T} P_{m}+P_{m} A-C^{T} L_{m}^{T} P_{m}+R_{m}+R_{1 m}+R_{2 m}+\left(\tau_{2}-\tau_{1}\right) Q_{m}+\alpha_{1} L_{f}^{2}+\alpha_{2} L_{f, d}^{2}+\beta_{1} L_{g m}^{2}+\beta_{2} L_{g m, d}^{2}, \\
& \zeta_{11, s}=A^{T} P_{s}+P_{s} A-C^{T} L_{s}^{T} P_{s}+R_{s}+R_{1 s}+R_{2 s}+\left(\tau_{2}-\tau_{1}\right) Q_{s}+\alpha_{3} L_{f}^{2}+\alpha_{4} L_{f, d}^{2}+\beta_{3} L_{g s}^{2}+\beta_{4} L_{g s, d}^{2}, \\
& \zeta_{11, o}=A^{T} P_{o}+P_{o} A-P_{o} B^{T} F^{T}-P_{o} B F+\left(\tau_{2}-\tau_{1}\right) Q_{o}+R_{o}+R_{1 o}+R_{2 o}+\alpha_{5} L_{f}^{2}+\alpha_{6} L_{f, d}^{2} .
\end{aligned}
$$


Proof. The balanced state for synchronization of $(\mathrm{M})$ and $(\mathrm{S})$ modules is adopted as follows. By considering $\widetilde{\theta}_{m}(t)=\theta_{m}-$ $\widehat{\theta}_{m}(t)$ and $\hat{\theta}_{m}(t-\tau)=\theta_{m}-\hat{\theta}_{m}(t-\tau)$ and assuming $g_{m}\left(x_{m}(t)\right)=B g\left(x_{m}(t)\right) \theta_{m}, \quad g_{s}\left(x_{s}(t)\right)=B g\left(x_{s}(t)\right) \theta_{s}$, $g_{d m}\left(x_{m}(t-\tau)\right)=B g\left(x_{m}(t-\tau)\right) \theta_{d m}$, and $g_{d s}\left(x_{s}(t-\tau)\right)=$ $B g\left(x_{s}(t-\tau)\right) \theta_{d s}$ and further utilizing some mathematical manipulation, we can derive

$$
\begin{aligned}
B g\left(x_{m}(t)\right) \theta_{m}-B g\left(\widehat{x}_{m}(t)\right) \hat{\theta}_{m}(t) & =g_{m}\left(x_{m}(t)\right)-g_{m}\left(\widehat{x}_{m}(t)\right)+B g\left(\widehat{x}_{m}(t)\right) \tilde{\theta}_{m}(t), \\
B g\left(x_{m}(t-\tau)\right) \theta_{m}-B g\left(\widehat{x}_{m}(t-\tau)\right) \hat{\theta}_{m}(t-\tau) & =g_{d m}\left(x_{m}(t-\tau)\right)-g_{d m}\left(\hat{x}_{m}(t-\tau)\right)+B g\left(\widehat{x}_{m}(t-\tau)\right) \tilde{\theta}_{m}(t-\tau), \\
B g\left(\hat{x}_{s}(t)\right) \hat{\theta}_{s}(t) & =g_{s}\left(x_{s}(t)\right)-g_{s}\left(\widehat{x}_{s}(t)\right)+B g\left(\hat{x}_{s}(t)\right) \tilde{\theta}_{s}(t), \\
B g\left(x_{s}(t-\tau)\right) \theta_{s}-B g\left(\hat{x}_{s}(t-\tau)\right) \hat{\theta}_{s}(t-\tau) & =g_{d s}\left(x_{s}(t-\tau)\right)-g_{d s}\left(\widehat{x}_{s}(t-\tau)\right)+B g\left(\widehat{x}_{s}(t-\tau)\right) \tilde{\theta}_{s}(t-\tau) .
\end{aligned}
$$

By letting

we obtain from equation (8)

$$
\begin{aligned}
\widetilde{\psi}_{g, m \hat{m}}(t) & =g_{m}^{T}\left(x_{m}(t)\right)-g_{m}^{T}\left(\widehat{x}_{m}(t)\right), \\
\widetilde{\psi}_{g, \widehat{s s}}(t) & =g_{s}^{T}\left(x_{s}(t)\right)-g_{s}^{T}\left(\widehat{x}_{s}(t)\right), \\
\widetilde{\psi}_{(g, d), m \hat{m}}(t, \tau) & =g_{d m}\left(x_{m}(t-\tau)\right)-g_{d m}\left(\widehat{x}_{m}(t-\tau)\right), \\
\widetilde{\psi}_{(g, d), \widehat{s s}}(t, \tau) & =g_{d s}\left(x_{s}(t-\tau)\right)-g_{d s}\left(\widehat{x}_{s}(t-\tau)\right),
\end{aligned}
$$

$$
\begin{aligned}
\dot{e}_{m}(t)= & A e_{m}(t)+A_{d} e_{m}(t-\tau)+\psi_{m}(t)+\psi_{d m}(t)+\widetilde{\psi}_{g, m \hat{m}}(t)+\widetilde{\psi}_{(g, d), m \hat{m}}(t, \tau) \\
& +B g\left(\widehat{x}_{m}(t)\right) \widetilde{\theta}_{m}(t)+B g\left(\widehat{x}_{m}(t-\tau)\right) \widetilde{\theta}_{m}(t-\tau) \\
& -L_{m} C e_{m}(t)+d_{m}+\frac{1}{2} B F e_{o}(t) .
\end{aligned}
$$

Thus, it is certain to acquire from equation (9)

$$
\begin{aligned}
\dot{e}_{s}(t)= & A e_{s}(t)+A_{d} e_{s}(t-\tau)+\psi_{s}(t)+\psi_{d s}(t)+\widetilde{\psi}_{g, \widehat{s s}}(t) \\
& +\widetilde{\psi}_{(g, d), \widehat{s s}}(t, \tau)+B g\left(\widehat{x}_{s}(t)\right) \tilde{\theta}_{s}(t)+B g\left(\widehat{x}_{s}(t-\tau)\right) \tilde{\theta}_{s}(t-\tau) \\
& -L_{s} C e_{s}(t)-\frac{1}{2} B F e_{o}(t)+d_{s}-B u_{g}(t),
\end{aligned}
$$


6

Complexity

Using equation (11), we have

$$
\begin{aligned}
\dot{e}_{o}(t)= & (A-B F) e_{o}(t)+A_{d} e_{o}(t-\tau)+\psi_{o}(t) \\
& +\psi_{d o}(t)+L_{m} C e_{m}(t)-L_{s} C e_{s}(t) .
\end{aligned}
$$

With the help of the Lyapunov function, the theorem is a proof that

$$
\begin{aligned}
& V(t, e)=e_{m}^{T}(t) P_{m} e_{m}(t)+\int_{-\tau_{2}}^{-\tau_{1}} \int_{t+\beta}^{t} e_{m}^{T}(\alpha) Q_{m} e_{m}(\alpha) d \alpha d \beta+\int_{-\tau}^{0} e_{m}^{T}(t+\sigma) R_{m} \\
& \times e_{m}(t+\sigma) d \sigma+\int_{-\tau_{1}}^{0} e_{m}^{T}(t+\sigma) R_{1 m} e_{m}(t+\sigma) d \sigma+\int_{-\tau_{2}}^{0} e_{m}^{T}(t+\sigma) R_{2 m} \\
& \times e_{m}(t+\sigma) d \sigma+\left[\int_{-\tau_{2}}^{-\tau_{1}} e_{m}(t+\sigma) d \sigma\right]^{T} W_{m}\left[\int_{-\tau_{2}}^{-\tau_{1}} e_{m}(t+\sigma) d \sigma\right] \\
& +e_{s}^{T}(t) P_{s} e_{s}(t)+\int_{-\tau_{2}}^{-\tau_{1}} \int_{t+\beta}^{t} e_{s}^{T}(\alpha) Q_{s} e_{s}(\alpha) d \alpha d \beta+\int_{-\tau}^{0} e_{s}^{T}(t+\sigma) R_{s} \\
& \times e_{s}(t+\sigma) d \sigma+\int_{-\tau_{1}}^{0} e_{s}^{T}(t+\sigma) R_{1 s} e_{s}(t+\sigma) d \sigma+\int_{-\tau_{2}}^{0} e_{s}^{T}(t+\sigma) R_{2 s} \\
& \times e_{s}(t+\sigma) d \sigma+\left[\int_{-\tau_{2}}^{-\tau_{1}} e_{s}(t+\sigma) d \sigma\right]^{T} W_{s}\left[\int_{-\tau_{2}}^{-\tau_{1}} e_{s}(t+\sigma) d \sigma\right] \\
& +e_{o}^{T}(t) P_{o} e_{o}(t)+\int_{-\tau_{2}}^{-\tau_{1}} \int_{t+\beta}^{t} e_{o}^{T}(\alpha) Q_{o} e_{o}(\alpha) d \alpha d \beta+\int_{-\tau}^{0} e_{o}^{T}(t+\sigma) R_{o} \\
& \times e_{o}(t+\sigma) d \sigma+\int_{-\tau_{1}}^{0} e_{o}^{T}(t+\sigma) R_{1 o} e_{o}(t+\sigma) d \sigma+\int_{-\tau_{2}}^{0} e_{o}^{T}(t+\sigma) R_{2 o} \\
& \times e_{o}(t+\sigma) d \sigma+\left[\int_{-\tau_{2}}^{-\tau_{1}} e_{o}(t+\sigma) d \sigma\right]^{T} W_{o}\left[\int_{-\tau_{2}}^{-\tau_{1}} e_{o}(t+\sigma) d \sigma\right] \\
& +\tilde{\theta}_{m}^{T}(t) \Theta_{m}^{-1} \widetilde{\theta}_{m}(t)+\tilde{\theta}_{s}^{T}(t) \Theta_{s}^{-1} \widetilde{\theta}_{s}(t)+ \\
& \tilde{\theta}_{m, d}^{T}(t-\tau) \Theta_{m, d}^{-1} \widetilde{\theta}_{m, d}(t-\tau)+\widetilde{\theta}_{s, d}^{T}(t-\tau) \Theta_{s, d}^{-1} \widetilde{\theta}_{s, d}(t-\tau) .
\end{aligned}
$$


Taking the time derivative of the Lyapunov energy function, equation (15) can be written as

$$
\begin{aligned}
& \dot{V}(t, e)=\dot{e}_{m}^{T}(t) P_{m} e(t)+e_{m}^{T}(t) P_{m} \dot{e}_{m}(t)+\left(\tau_{2}-\tau_{1}\right) e_{m}^{T}(t) Q e_{m}(t)-\int_{t-\tau_{2}}^{t-\tau_{1}} e_{m}^{T}(\sigma) \\
& \times Q e_{m}(\sigma) d \sigma+e_{m}^{T}(t) R e_{m}(t)-(1-\dot{\tau}) e_{m}^{T}(t-\tau) R e_{m}(t-\tau)+e_{m}^{T}(t) R_{1} e_{m}(t) \\
& -e_{m}^{T}\left(t-\tau_{1}\right) R_{1} e_{m}\left(t-\tau_{1}\right)+e_{m}^{T}(t) R_{2} e_{m}(t)-e_{m}^{T}\left(t-\tau_{2}\right) R_{2} e_{m}\left(t-\tau_{2}\right) \\
& +\left[e_{m}\left(t-\tau_{1}\right)-e_{m}\left(t-\tau_{2}\right)\right]^{T} W\left[\int_{t-\tau_{2}}^{t-\tau_{1}} e_{m}(\sigma) d \sigma\right]+\left[\int_{t-\tau_{2}}^{t-\tau_{1}} e_{m}(\sigma) d \sigma\right]^{T} \\
& \times W\left[e_{m}\left(t-\tau_{1}\right)-e_{m}\left(t-\tau_{2}\right)\right] \\
& \dot{e}_{s}^{T}(t) P_{s} e(t)+e_{s}^{T}(t) P_{s} \dot{e}_{s}(t)+\left(\tau_{2}-\tau_{1}\right) e_{s}^{T}(t) Q e_{s}(t)-\int_{t-\tau_{2}}^{t-\tau_{1}} e_{s}^{T}(\sigma) \\
& \times Q e_{s}(\sigma) d \sigma+e_{s}^{T}(t) R e_{s}(t)-(1-\dot{\tau}) e_{s}^{T}(t-\tau) R e_{s}(t-\tau)+e_{s}^{T}(t) R_{1} e_{s}(t) \\
& -e_{s}^{T}\left(t-\tau_{1}\right) R_{1} e_{s}\left(t-\tau_{1}\right)+e_{s}^{T}(t) R_{2} e_{s}(t)-e_{s}^{T}\left(t-\tau_{2}\right) R_{2} e_{s}\left(t-\tau_{2}\right) \\
& +\left[e_{s}\left(t-\tau_{1}\right)-e_{s}\left(t-\tau_{1}\right)\right]^{T} W\left[\int_{t-\tau_{2}}^{t-\tau_{1}} e_{s}(\sigma) d \sigma\right]+\left[\int_{t-\tau_{2}}^{t-\tau_{1}} e_{s}(\sigma) d \sigma\right]^{T} \\
& \times W\left[e_{s}\left(t-\tau_{1}\right)-e_{s}\left(t-\tau_{1}\right)\right] \\
& \dot{e}_{o}^{T}(t) P_{o} e(t)+e_{o}^{T}(t) P_{o} \dot{e}_{o}(t)+\left(\tau_{2}-\tau_{1}\right) e_{o}^{T}(t) Q e_{o}(t)-\int_{t-\tau_{2}}^{t-\tau_{1}} e_{o}^{T}(\sigma) \\
& \times Q e_{o}(\sigma) d \sigma+e_{o}^{T}(t) R e_{o}(t)-(1-\dot{\tau}) e_{o}^{T}(t-\tau) R e_{o}(t-\tau)+e_{o}^{T}(t) R_{1} e_{o}(t) \\
& -e_{o}^{T}\left(t-\tau_{1}\right) R_{1} e_{o}\left(t-\tau_{1}\right)+e_{o}^{T}(t) R_{2} e_{o}(t)-e_{o}^{T}\left(t-\tau_{2}\right) R_{2} e_{o}\left(t-\tau_{2}\right) \\
& +\left[e_{o}\left(t-\tau_{1}\right)-e_{o}\left(t-\tau_{2}\right)\right]^{T} W\left[\int_{t-\tau_{2}}^{t-\tau_{1}} e_{o}(\sigma) d \sigma\right]+\left[\int_{t-\tau_{2}}^{t-\tau_{1}} e_{o}(\sigma) d \sigma\right]^{T} \\
& \times W\left[e_{o}\left(t-\tau_{1}\right)-e_{o}\left(t-\tau_{2}\right)\right] \\
& +2 \tilde{\theta}_{m}^{T}(t) \Theta_{m}^{-1} \dot{\tilde{\theta}}_{m}(t)+2 \tilde{\theta}_{s}^{T}(t) \Theta_{s}^{-1} \dot{\tilde{\theta}}_{s}(t) \\
& +2 \widetilde{\theta}_{m, d}^{T}(t-\tau) \Theta_{m, d}^{-1} \dot{\tilde{\theta}}_{m, d}(t-\tau)+2 \tilde{\theta}_{s, d}^{T}(t-\tau) \Theta_{s, d}^{-1} \dot{\tilde{\theta}}_{s, d}(t-\tau) .
\end{aligned}
$$

Assume $d_{m}=d_{s}=0$.

From equation (21), clearly, $\mathrm{V}(t)<0$ is guaranteed if $1<0$ is fulfilled. Thus, the em $(\mathrm{t})$, es $(\mathrm{t})$, and eo(t) error signals are asymptotically steady. Accordingly, the (M)-(S) systems in equations (1) and (2) are synchronized, which concludes the proof.
Theorem 1. It gives the result of the synchronization problem for the (M)-(S) nonlinear systems with the requirement that $F$ is the controller gain matrix and Lm and Ls are the observer gain matrices. To remove this restriction, we have suggested a solution for the approximation values of $\mathrm{Lm}, \mathrm{Ls}$, and $\mathrm{F}$, using a convex routine solution. Now, we set out 
a methodology for solving the inequality matrix with the help of the two-step LMI-based approach:

$$
\begin{aligned}
& \Phi_{11}=\left[\begin{array}{ccccccccc}
\zeta_{11, m} & A_{d} P_{m} & 0 & 0 & 0 & 0 & 0 & 0 & 0 \\
A_{d}^{T} P_{m} & -(1-\tau) R & 0 & 0 & 0 & 0 & 0 & 0 & 0 \\
* & * & -R_{1 m} & 0 & W_{m} & 0 & 0 & 0 & 0 \\
* & * & * & -R_{2 m} & -W_{m} & 0 & 0 & 0 & 0 \\
* & * & W_{m} & -W_{m} & -\frac{1}{\tau_{2}-\tau_{1}} Q_{m} & 0 & 0 & 0 & 0 \\
* & * & * & * & * & \zeta_{11, s} & A_{d} P_{s} & 0 & 0 \\
* & * & * & * & * & A_{d}^{T} P_{s} & -(1-\tau) R & 0 & 0 \\
* & * & * & * & * & * & * & -R_{1 s} & 0 \\
* & * & * & * & * & * & * & * & -R_{2 s}
\end{array}\right] \text {, } \\
& \Phi_{22}=\left[\begin{array}{ccccccccc}
-\frac{1}{\tau_{2}-\tau_{1}} Q_{s} & 0 & 0 & 0 & 0 & 0 & 0 & 0 & 0 \\
* & \zeta_{11, o} & A_{d} P_{o} & 0 & 0 & 0 & 0 & 0 & 0 \\
* & A_{d}^{T} P_{o} & -(1-\tau) R & 0 & 0 & 0 & 0 & 0 & 0 \\
* & * & * & -R_{1 o} & 0 & W_{o} & 0 & 0 & 0 \\
* & * & * & * & -R_{2 o} & -W_{o} & 0 & 0 & 0 \\
* & * & * & W_{o} & -W_{o}-\frac{1}{\tau_{2}-\tau_{1}} Q_{0} & 0 & 0 & 0 \\
* & * & * & * & * & * & -\alpha & & \\
* & * & * & * & * & * & * & -\alpha_{2} I_{n} & 0 \\
* & * & * & * & * & * & * & * & -\beta_{1} I_{n}
\end{array}\right], \\
& \Phi_{33}=\left[\begin{array}{ccccccc}
-\beta_{2} I_{n} & 0 & 0 & 0 & 0 & 0 & 0 \\
* & -\alpha_{3} I_{n} & 0 & 0 & 0 & 0 & 0 \\
* & * & -\alpha_{4} I_{n} & 0 & 0 & 0 & 0 \\
* & * & * & -\beta_{3} I_{n} & 0 & 0 & 0 \\
* & * & * & * & -\beta_{4} I_{n} & 0 & 0 \\
* & * & * & * & * & -\alpha_{5} I_{n} & 0 \\
* & * & * & * & * & * & -\alpha_{6} I_{n}
\end{array}\right],
\end{aligned}
$$


$\Phi_{12}=\left[\begin{array}{ccccccccc}0 & 0.5 P_{m} B F+L_{m}^{T} C^{T} P_{o} & 0 & 0 & 0 & 0 & P_{m} & P_{m} & P_{m} \\ 0 & 0 & 0 & 0 & 0 & 0 & 0 & 0 & 0 \\ 0 & 0 & 0 & 0 & 0 & 0 & 0 & 0 & 0 \\ 0 & 0 & 0 & 0 & 0 & 0 & 0 & 0 & 0 \\ 0 & 0 & 0 & 0 & 0 & 0 & 0 & 0 & 0 \\ 0 & 0.5 P_{s} B F-L_{s}^{T} C^{T} P_{o} & 0 & 0 & 0 & 0 & 0 & 0 & 0 \\ 0 & 0 & 0 & 0 & 0 & 0 & 0 & 0 & 0 \\ W_{s} & 0 & 0 & 0 & 0 & 0 & 0 & 0 & 0 \\ -W_{s} & 0 & 0 & 0 & 0 & 0 & 0 & 0 & 0\end{array}\right]$,

$\Phi_{13}=\left[\begin{array}{ccccccc}P_{m} & 0 & 0 & 0 & 0 & 0 & 0 \\ 0 & 0 & 0 & 0 & 0 & 0 & 0 \\ 0 & 0 & 0 & 0 & 0 & 0 & 0 \\ 0 & 0 & 0 & 0 & 0 & 0 & 0 \\ 0 & 0 & 0 & 0 & 0 & 0 & 0 \\ 0 & P_{s} & P_{s} & P_{s} & P_{s} & 0 & 0 \\ 0 & 0 & 0 & 0 & 0 & 0 & 0 \\ 0 & 0 & 0 & 0 & 0 & 0 & 0 \\ 0 & 0 & 0 & 0 & 0 & 0 & 0\end{array}\right]$,

$\Phi_{21}=\left[\begin{array}{ccccccccc}* & * & * & * & * & * & * & W_{s} & -W_{s} \\ 0.5 P_{m} B^{T} F^{T}+L_{m} C P_{o} & * & * & * & * & 0.5 P_{m} B^{T} F^{T}+L_{s} C P_{o} & * & * & * \\ * & * & * & * & * & * & * & * & * \\ * & * & * & * & * & * & * & * & * \\ * & * & * & * & * & * & * & * & * \\ * & * & * & * & * & * & * & * & * \\ P_{m} & * & * & * & * & * & * & * & * \\ P_{m} & * & * & * & * & * & * & * & * \\ P_{m} & * & * & * & * & * & * & * & *\end{array}\right]$, 


$$
\begin{aligned}
& \Phi_{23}=\left[\begin{array}{ccccccc}
0 & 0 & 0 & 0 & 0 & 0 & 0 \\
0 & 0 & 0 & 0 & 0 & P_{o} & P_{o} \\
0 & 0 & 0 & 0 & 0 & 0 & 0 \\
0 & 0 & 0 & 0 & 0 & 0 & 0 \\
0 & 0 & 0 & 0 & 0 & 0 & 0 \\
0 & 0 & 0 & 0 & 0 & 0 & 0 \\
0 & 0 & 0 & 0 & 0 & 0 & 0 \\
0 & 0 & 0 & 0 & 0 & 0 & 0 \\
0 & 0 & 0 & 0 & 0 & 0 & 0
\end{array}\right], \\
& \Phi_{31}=\left[\begin{array}{ccccccccc}
P_{m} & * & * & * & * & * & * & * & * \\
* & * & * & * & * & P_{s} & * & * & * \\
* & * & * & * & * & P_{s} & * & * & * \\
* & * & * & * & * & P_{s} & * & * & * \\
* & * & * & * & * & P_{s} & * & * & * \\
* & * & * & * & * & * & * & * & * \\
* & * & * & * & * & * & * & * & *
\end{array}\right] \\
& \Phi_{32}=\left[\begin{array}{lllllllll}
* & * & * & * & * & * & * & * & * \\
* & * & * & * & * & * & * & * & * \\
* & * & * & * & * & * & * & * & * \\
* & * & * & * & * & * & * & * & * \\
* & * & * & * & * & * & * & * & * \\
* & P_{o} & * & * & * & * & * & * & * \\
* & P_{o} & * & * & * & * & * & * & *
\end{array}\right]
\end{aligned}
$$

\section{Simulation and Results}

Simulation of the suggested methods for the synchronization of the (M) and (S) systems with undefined parameters, as planned in Theorem 1, is shown in the accompanying simulation outcomes for FHN (FitzHugh-Nagumo) (M)-(S) designs. The suggested methodology was completed with the help of simulation work using MATLAB software and FHN numerical models. FHN is generally utilized in genetic systems, such as brain stimulation therapy, considering the performance of neurons in electricity. It helps in investigating symptoms and diseases of the brain, including tremors resulting from disorders of the brain's neurons. This kind of infection occurs in various parts of the brain. The FHN system is defined below:

$$
\begin{aligned}
\dot{X}_{m 1} & =\left(X_{m 1}^{2}-X_{m 1}\right)\left(1-R_{1} X_{m 1}\right)-X_{m 2}+I_{o} \dot{X}_{m 2}=B X_{m 2}, \\
\dot{X}_{s i} & =\left(X_{s i}^{2}-X_{s i}\right)\left(1-R_{i} X_{s i}\right)-X_{s i}+I_{o} X_{s i+1}=B X_{s i+1}, \\
I_{o} & =\left(\frac{m}{\omega}\right) \operatorname{Cos}(\omega t),
\end{aligned}
$$

where $I_{o}$ is the current in the above equation, $m=0.099, \omega$ $=2 \pi f$, and $f=0.128$. Chaotic systems are sensitive to initial conditions. By changing the value of initial conditions, the phase portrait, i.e., behaviour of nonlinear chaotic systems, changes. The initial conditions for the (M) and (S) systems are $X_{s 1(0)}=0.399$ and $X_{s i+1(0)}=0.099$. The other parameters are $B=1.01, R_{1}=10.09$, and $R_{2}=9.89$.

The phasor picture and individual reactions to the nonlinear chaotic performance of the (M)-(S) FHN system are exposed in Figure 1. For the (M)-(S) systems, various initial conditions are used. Different error signals are designed between the master system with its observer and the slave system with its observer and introduced in Figures 2(a) and 2(b), for Theorem 1, respectively. Figure 3 describes the error signal between the $(M)$ observer state and the (S) observer state. The simulation results are given for a nonadaptive control strategy under three different conditions. First, neuron behaviour with the help of the FHN system is generalized. In neurons, membrane potential is not the same in all living beings. It can be standardized using methods for an alternative scaling factor, so this is pertinent for all types of neurons. Second, the numerical articulations of the FHN system are, for the most part, dependent on the ordinary membrane potential. Third, standardized potential usage gain matrices must be controlled to finally synchronize (M)-(S) systems.

The FHN model is related to the matrices, as indicated by the nonlinear chaotic master and slave systems: 

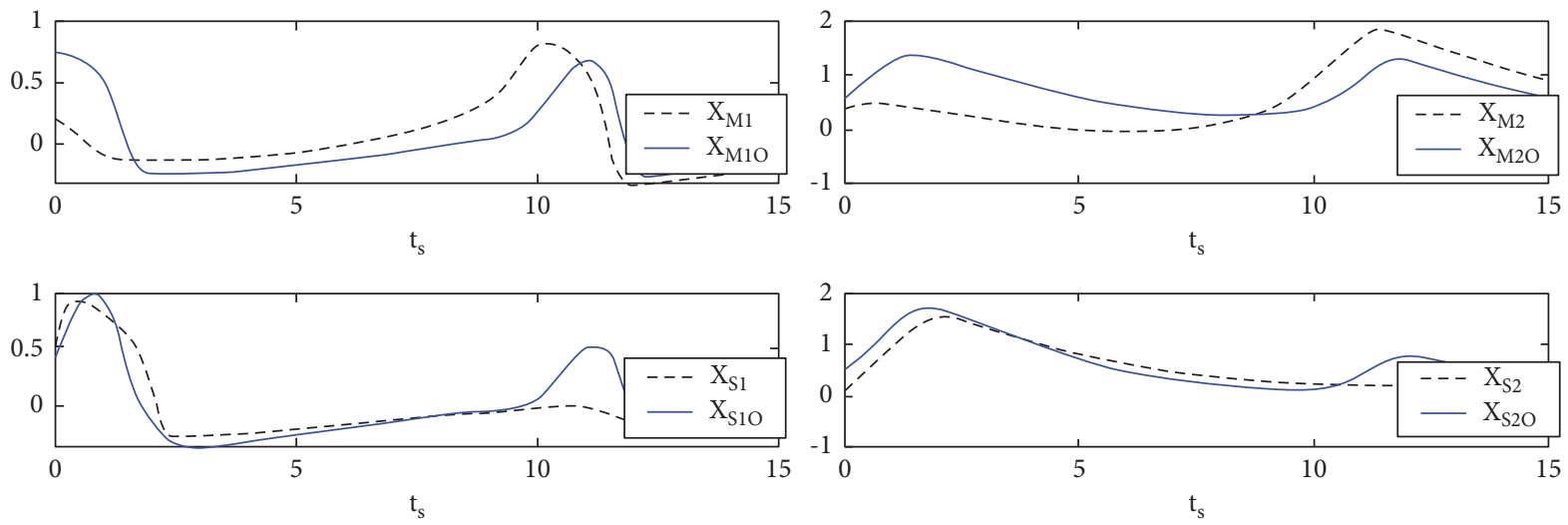

(a)
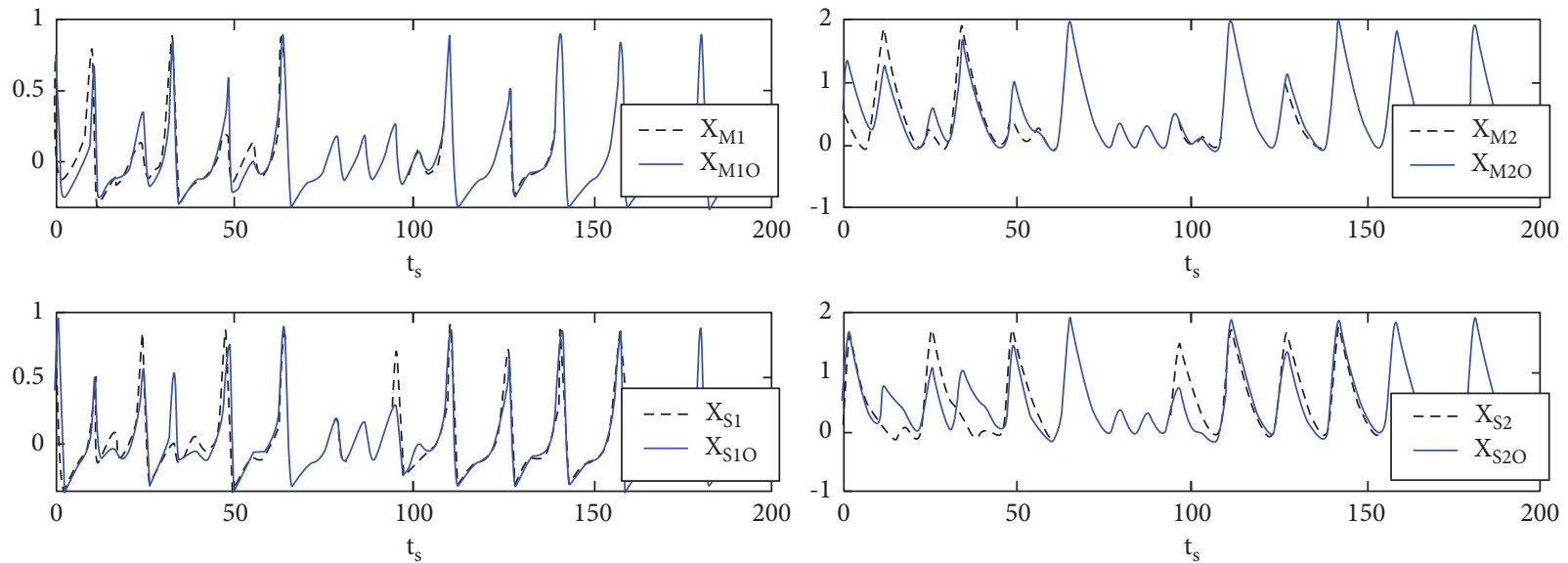

(b)

FIgURE 1: (a) Standardized potential of the master and (b) four slave systems and their respective observers of Theorem 1.

$$
\begin{aligned}
S_{i} & =\left[\begin{array}{rr}
-1.00 & -1.00 \\
1.00 & 0.00
\end{array}\right], \\
X & =\left[\begin{array}{l}
1.00 \\
0.00
\end{array}\right], \\
Y & =\left[\begin{array}{l}
1.00 \\
0.00
\end{array}\right], \\
F(x(t)) & =\left[\begin{array}{c}
11.00 x_{1}{ }^{2}+10.00 x_{1}{ }^{3}+\left(\frac{m}{\omega}\right) \operatorname{Cos}(\omega t) \\
0.00
\end{array}\right] .
\end{aligned}
$$

By using the adaptive scheme, synchronizing the (M)-(S) systems according to Theorem $1, \mathrm{Lm}$ and $\mathrm{Ls}$ are the gain matrices for the observer's master and slave systems, respectively. By varying the values of these observers gain matrices Lm and Ls and control gain matrix F, the effectiveness and efficiency of proposed control methodology may vary. After some empirical analysis, the values of the observer gain matrices and controller gain matrix are chosen. These Lm, Ls, and F values are chosen as follows:

$$
\begin{aligned}
L_{m} & =\left[\begin{array}{l}
1.32 \\
0.00
\end{array}\right], \\
L_{s i} & =\left[\begin{array}{l}
1.32 \\
0.00
\end{array}\right], \\
F & =[1.000 .00], \\
\xi & =F\left(\widehat{x}_{m}(t)-\widehat{x}_{s i}(t)\right) .
\end{aligned}
$$

Figure 4 represents the result of the controller. In equation (26), $\xi$ is the controlling function, which controls the behaviour of the (M)-(S) system. Theorem 1 illustrates this in Figure 1, which shows the standardized potential of the (M)-(S) system with its observers. In Figure 5, Theorem 1 illustrates the observer recovery variables for the (M)-(S) systems. Figure 6 explains the error signals between the (M) system and its master observer and between the (S) system and its slave observer. Lm and Ls are the gain matrices for the observer's respective master and slave systems, potentially influencing the master error em $(\mathrm{t})$ and slave error es $(\mathrm{t})$. " $F$ " can clearly affect eoi(t). In the unlikely event where we 


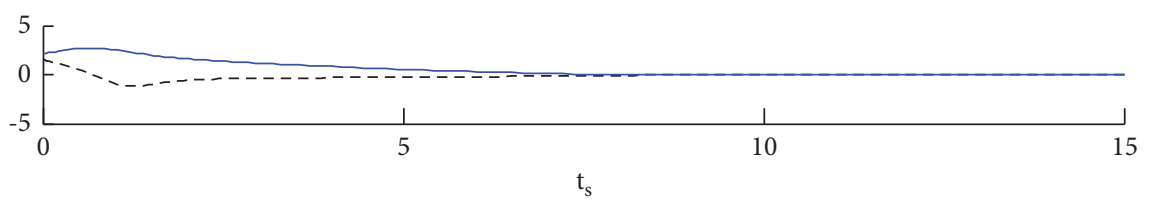

$$
--\mathrm{e}_{1}
$$

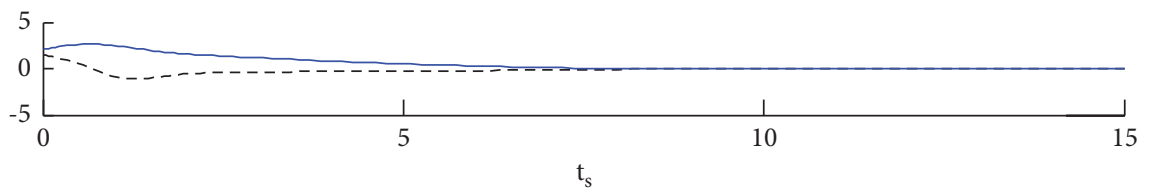

$---\mathrm{e}_{3}$

(a)

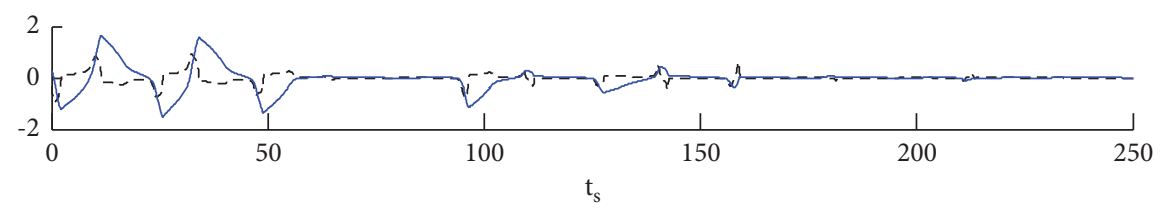

$---\mathrm{e}_{1}$

$-\mathrm{e}_{2}$

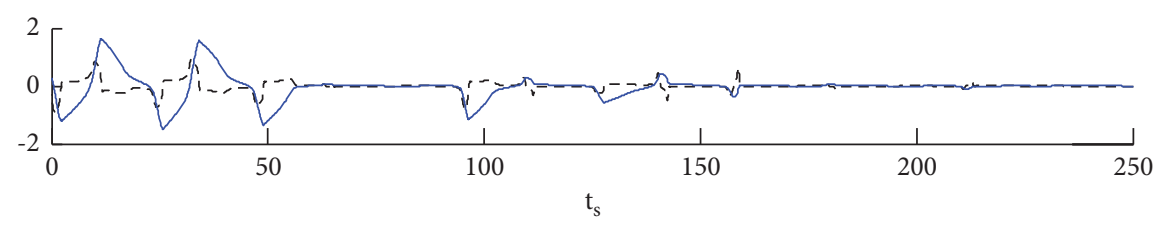

$---\mathrm{e}_{3}$

$-\mathrm{e}_{4}$

(b)

Figure 2: (a) Error between the states of the master and (b) the slave system for Theorem 1.

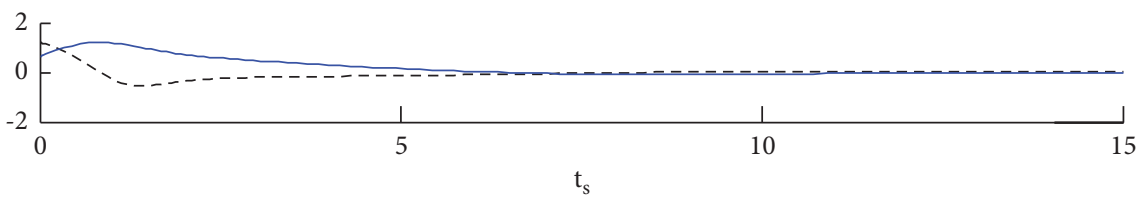

$---\mathrm{e}_{\mathrm{ol}}$
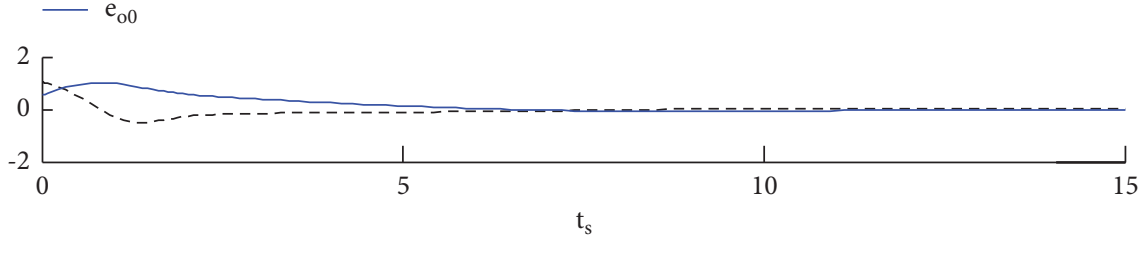

$--\mathrm{e}_{\mathrm{o} 2}$

(a)

Figure 3: Continued. 

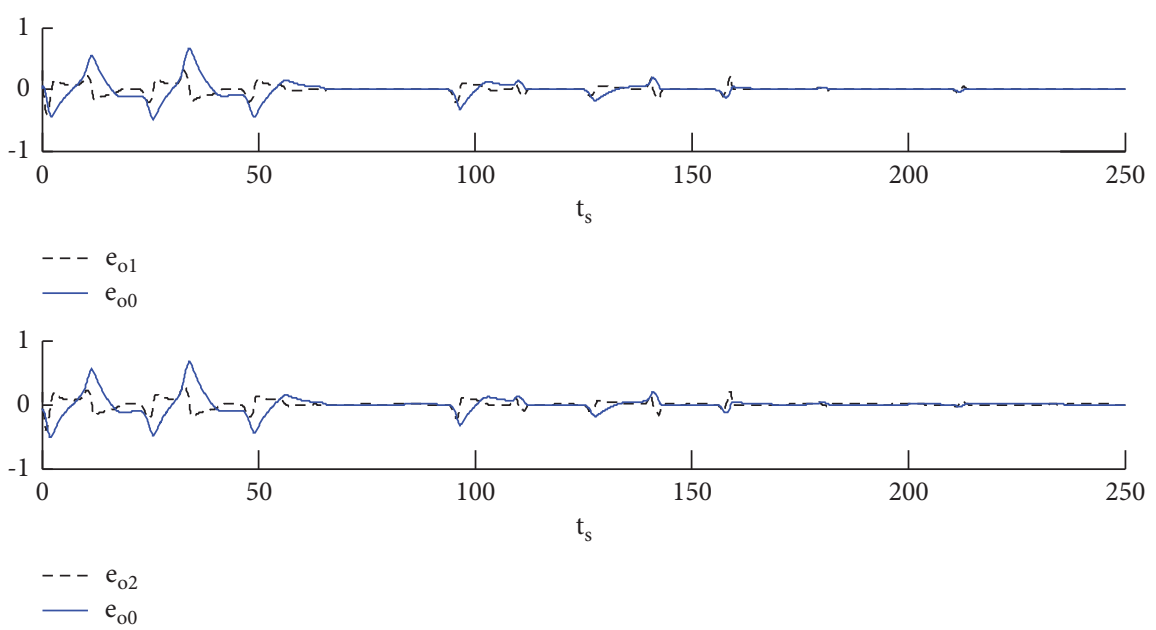

(b)

Figure 3: (a) Error between the master observer states and (b) that corresponding to the states of slave observers of Theorem 1.
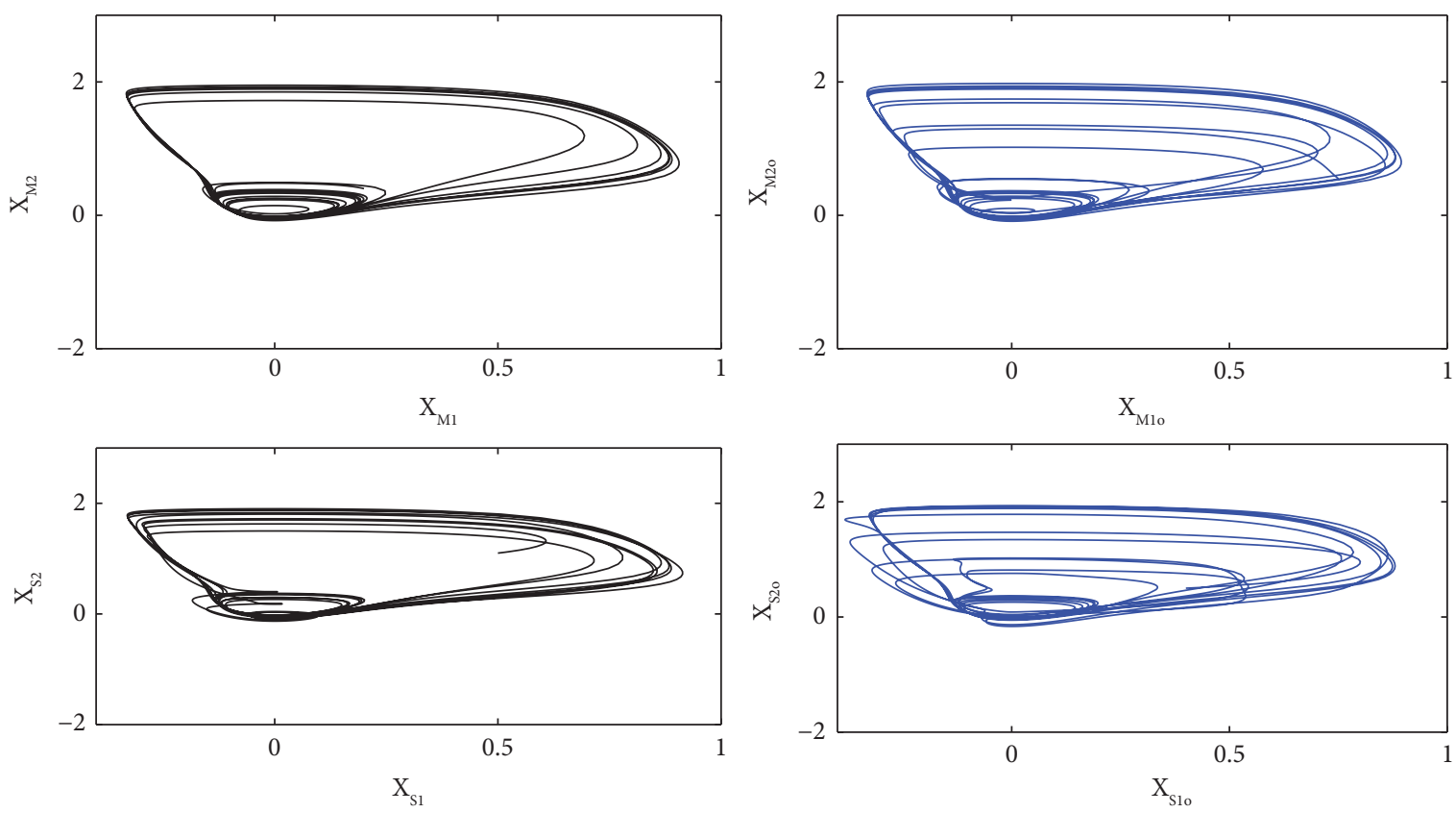

Figure 4: Phase portraits of master and slave systems of Theorem 1.

change the gain matrices' standards, these straightforwardly influence the synchronizing time. In Figure 2, Theorem 1 shows the error signals between master and slave states. Finally, Figure 3 describes the error signals between the (M) observer state and $(\mathrm{S})$ observer states.

For the representation of the degree of synchronization statistically [31, 32], error-based DOS criteria are defined as follows:

$$
\operatorname{DOS}(\widetilde{e})=1-\frac{\tilde{e}_{2}}{\widetilde{e}_{2, \max }\left(\sqrt{\int_{0}^{t} \widetilde{e}^{2} d t} /\left.\sqrt{\int_{0}^{t} \tilde{e}^{2} d t}\right|_{\text {max }}\right)},
$$

where $\widetilde{e}_{2}$ and $\widetilde{e}_{2, \max }$ are the 2-norm of error $\tilde{e}$ and the maximum value of the norm, respectively. Note that the minimum and maximum values of degree of synchronization $(\operatorname{DOS}(\widetilde{e}))$ are 0 and 1 , respectively. The maxima occurs for the minimum synchronization error, that is, $\widetilde{e}_{2}=0$. While the minima occurs for $\widetilde{e}_{2}=\widetilde{e}_{2, \max }$, when synchronization error is maximum. It is worth mentioning that the maximum value $\widetilde{e}_{2, \max }$ can be achieved by selecting either $L_{m}=L_{s}=\left[\begin{array}{ll}0 & 0\end{array}\right]^{T}$ for any particular value of $F$ or by utilitarian of $F=\left[\begin{array}{ll}0 & 0\end{array}\right]$ with some fixed values of $L_{m}$ and $L_{s}$. Degree of synchronization is calculated for nonadaptive case to show the effect of variations in $L_{m}, L_{s}$, and $F$. Tables 1 and 


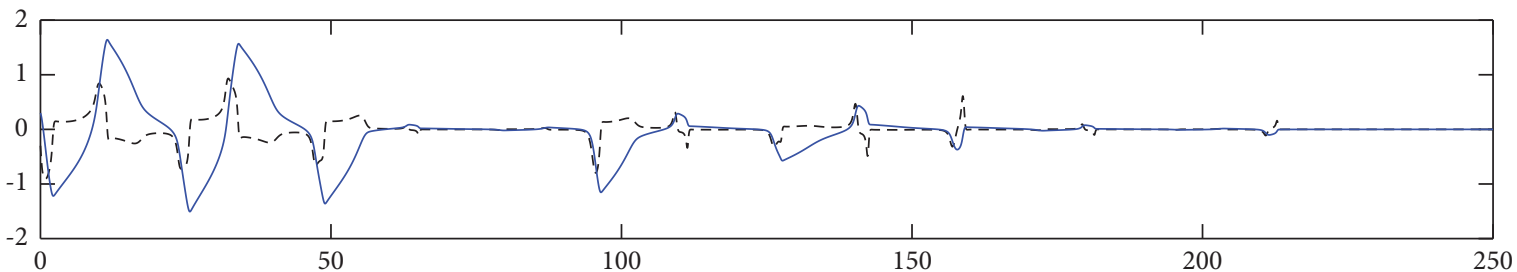

$---\mathrm{e}_{\mathrm{M} 1}$

(a)
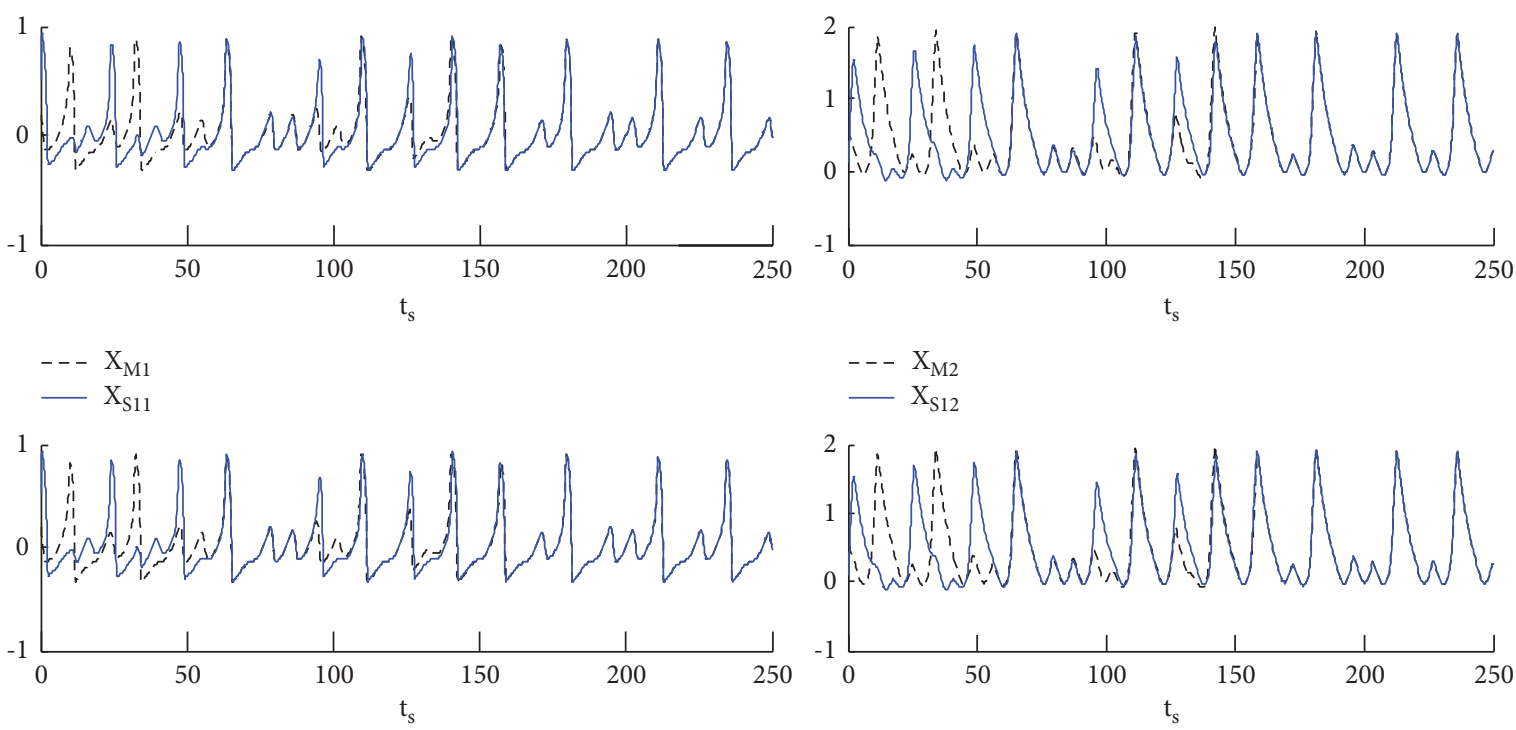

$---\mathrm{X}_{\mathrm{M} 2}$
$-\mathrm{X}_{\mathrm{S} 12}$

$---\mathrm{X}_{\mathrm{M} 1}$

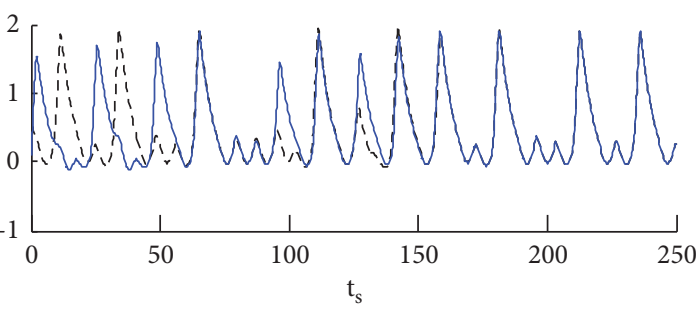

$-\mathrm{X}_{\mathrm{S} 21}$

$---\mathrm{X}_{\mathrm{M} 2}$

$-\mathrm{X}_{\mathrm{S} 22}$

(b)

FIgURe 5: (a) Master and slave systems and (b) their respective observer recovery variables of Theorem 1.

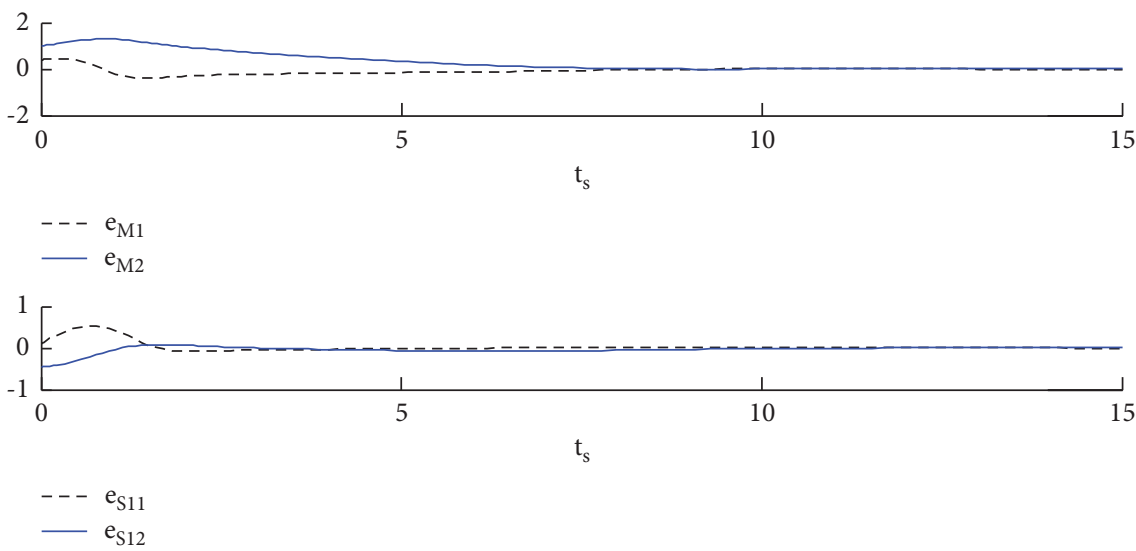

(a)

Figure 6: Continued. 


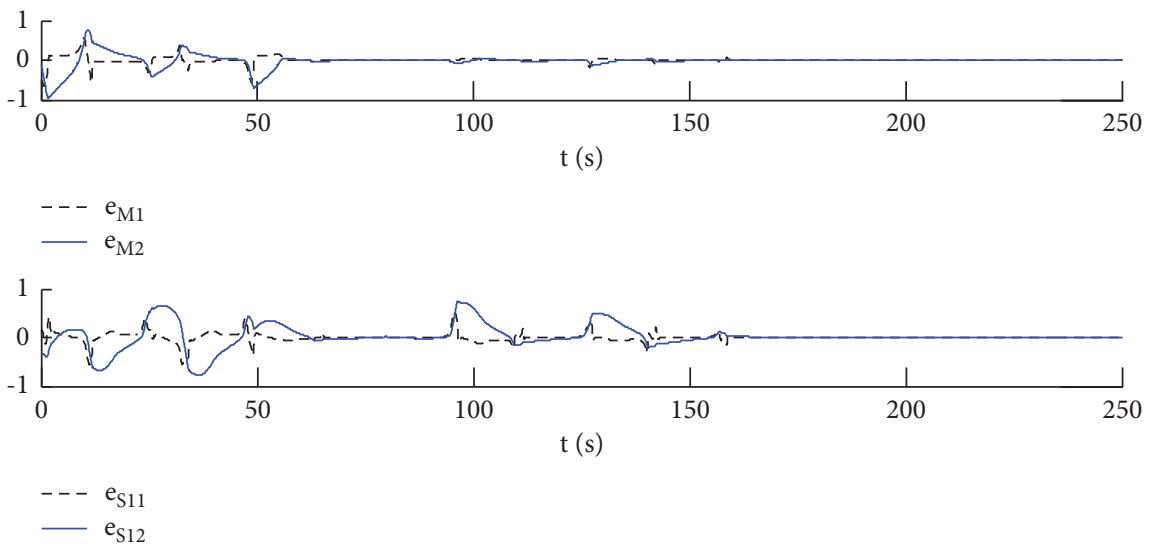

(b)

Figure 6: (a) Errors between the corresponding states of master and (b) master observer and slave observer for Theorem 1.

TABLE 1: DOS for different values of $\mathrm{Lm}=\mathrm{Ls}$ and fixed $F=\left[\begin{array}{ll}1 & 0\end{array}\right]$.

\begin{tabular}{lccc}
\hline Serial no. & $L_{m}=L_{\mathrm{s}}$ & $\left\|e_{m 1}\right\|$ & DoS \\
\hline 1 & {$\left[\begin{array}{lll}0 & 0\end{array}\right]^{T}$} & 31.5261 & 0 \\
2 & {$\left[\begin{array}{lll}0.1 & 0\end{array}\right]^{T}$} & 28.2900 & 0.102648 \\
3 & {$\left[\begin{array}{lll}0.14 & 0\end{array}\right]^{T}$} & 13.5245 & 0.5710063 \\
4 & {$\left[\begin{array}{lll}0.15 & 0\end{array}\right]^{T}$} & 8.4689 & 0.731369 \\
5 & {$\left[\begin{array}{ll}5 & 0\end{array}\right]^{T}$} & 0.0470 & 0.998509 \\
6 & {$\left[\begin{array}{lll}20 & 0\end{array}\right]^{T}$} & 0.0131 & 0.999584 \\
7 & {$\left[\begin{array}{lll}100 & 0\end{array}\right]^{T}$} & 0.0095 & 0.999699 \\
\hline
\end{tabular}

TABLE 2: DOS for different values of $L_{\mathrm{m}}=L_{\mathrm{s}}$ and fixed $F=\left[\begin{array}{ll}1.3 & 0\end{array}\right]^{\mathrm{T}}$.

\begin{tabular}{lccc}
\hline Serial no. & $L_{m}=L_{\mathrm{s}}$ & $\left\|e_{m 1}\right\|$ & DoS \\
\hline 1 & {$\left[\begin{array}{ll}0 & 0\end{array}\right]^{T}$} & 3.0379 & 0 \\
2 & {$\left[\begin{array}{ll}0.2 & 0\end{array}\right]^{T}$} & 2.4210 & 0.203068 \\
3 & {$\left[\begin{array}{ll}0.5 & 0\end{array}\right]^{T}$} & 0.8489 & 0.391389 \\
4 & {$\left[\begin{array}{ll}1 & 0\end{array}\right]^{T}$} & 0.4706 & 0.84509 \\
5 & {$\left[\begin{array}{ll}5 & 0\end{array}\right]^{T}$} & 0.1324 & 0.956417 \\
6 & {$\left[\begin{array}{lll}20 & 0\end{array}\right]^{T}$} & 0.0165 & 0.994569 \\
7 & 0.0052 & 0.0095 & 0.998288 \\
\hline
\end{tabular}

2 demonstrate the effect of $L_{m}=L_{s}$ and Fon the DOS, respectively. It can be concluded that increase in the entries of $L_{m}$ and $F$ can increase the degree of synchronization errors $e_{m 1}(t)$ and $e_{o 1}(t)$, respectively.

\section{Conclusion}

Synchronization of the two nonlinear systems, as well as chaotic frameworks with time delay, uncertainties, and disturbance, are recognized in this research study. A controller is designed utilizing the robust adaptive input control hypothesis. Along with the laws of adaptation for the approximation of boundaries, the planned delay rate-dependent controller ensures the synchronization of chaos, bringing synchronization errors to zero. The simulations using MATLAB confirm the adequacy of the proposed strategy. This is despite the fact that the model considered is for complex nonlinear chaotic framework with time delays with undefined elements. The result is also significant for its moderately simple, nonlinear frameworks with defined elements and consistent delays. As far as the future work is concerned, the distributed systems' synchronization of nonlinear systems can be considered. New methodologies can be sought for the distributed nonlinear systems having network delays with varying parameters.

\section{Data Availability}

The data used to support the findings of this study are available within the article. The raw data used to support the findings of this study are available from the corresponding author upon request.

\section{Conflicts of Interest}

The authors declare no conflicts of interest.

\section{Acknowledgments}

The authors would like to thank the Deanship of Scientific Research, Qassim University, for funding the publication of this project.

\section{References}

[1] T. L. Carroll, L. M. Pecora, and F. J. Rachford, "Chaotic transients and multiple attractors in spin-wave experiments," Physical Review Letters, vol. 59, no. 25, pp. 2891-2894, 1987.

[2] L. M. Pecora, "Derivation and generalization of the suhl spinwave instability relations," Physical Review B, vol. 37, no. 10, pp. 5473-5477, 1988.

[3] T. L. Carroll and L. M. Pecora, "Synchronizing chaotic circuits," IEEE Transactions on Circuits and Systems, vol. 38, no. 4, pp. 453-456, 1991.

[4] M. Chadli and I. Zelinka, "Chaos synchronization of unknown inputs Takagi-Sugeno fuzzy: application to secure communications," Computers \& Mathematics with Applications, vol. 68, no. 12, pp. 2142-2147, 2014. 
[5] J. M. G. Miranda, Synchronization and Control of Chaos: An Introduction for Scientists and Engineers, Imperial College Press, London, UK, 2004

[6] S. Beyhan, "Runge-Kutta model-based nonlinear observer for synchronization and control of chaotic systems," ISA Transactions, vol. 52, no. 4, pp. 501-509, 2013.

[7] M. T. Yassen, "Controlling chaos and synchronization for new chaotic system using linear feedback control," Chaos, Solitons \& Fractals, vol. 26, no. 3, pp. 913-920, 2005.

[8] M. Rehan and K. S. Hong, "Robust synchronization of delayed chaotic fitzhugh-nagumo neurons under external electrical stimulation," Computational and Mathematical Methods in Medicine, vol. 2021, Article ID 230980, 11 pages, 2012.

[9] M. H. Zaheer, M. Rehan, G. Mustafa, and M. Ashraf, "Delayrange-dependent chaos synchronization approach under varying time-lags and delayed nonlinear coupling," ISA Transactions, vol. 53, no. 6, pp. 1716-30, 2014.

[10] S. C. Jeong, D. H. Ji, J. H. Park, and S. C. Won, “Adaptive synchronization for uncertain chaotic neural networks with mixed time delays using fuzzy disturbance observer," Applied Mathematics and Computation, vol. 219, no. 11, pp. 59845995, 2013.

[11] C.-C. Yang, "Adaptive control and synchronization of identical new chaotic flows with unknown parameters via single input," Applied Mathematics and Computation, vol. 216, no. 4, pp. 1316-1324, 2010.

[12] C.-C. Yang, "Adaptive synchronization of Lü hyperchaotic system with uncertain parameters based on single-input controller," Nonlinear Dynamics, vol. 63, no. 3, pp. 447-454, 2011.

[13] A. Abdullah, "Synchronization and secure communication of uncertain chaotic systems based on full-order and reducedorder output-affine observers," Applied Mathematics and Computation, vol. 219, no. 19, pp. 10000-10011, 2013.

[14] J. Pena Ramirez, R. H. B. Fey, and H. Nijmeijer, "Synchronization of weakly nonlinear oscillators with Huygens' coupling," Chaos: An Interdisciplinary Journal of Nonlinear Science, vol. 23, no. 3, Article ID 033118, 2013.

[15] E. S. Kuetche Mbe, H. B. Fotsin, J. Kengne, and P. Woafo, "Parameters estimation based adaptive Generalized Projective Synchronization (GPS) of chaotic Chua's circuit with application to chaos communication by parametric modulation," Chaos, Solitons \& Fractals, vol. 61, pp. 27-37, 2014.

[16] J. Yang and F. Zhu, "Synchronization for chaotic systems and chaos-based secure communications via both reduced-order and step-by-step sliding mode observers," Communications in Nonlinear Science and Numerical Simulation, vol. 18, no. 4, pp. 926-937, 2013.

[17] B. Liu, L. Wang, Y.-H. Jin, D.-X. Huang, and F. Tang, "Control and synchronization of chaotic systems by differential evolution algorithm," Chaos, Solitons \& Fractals, vol. 34, no. 2, pp. 412-419, 2007.

[18] A. N. Njah, "Tracking control and synchronization of the new hyperchaotic liu system via backstepping techniques," Nonlinear Dynamics, vol. 61, no. 2, pp. 1-9, 2010.

[19] G. Hussain, M. Siddique, M. M. Hussain, M. T. Hassan, and N. Aslam, "Synchronization of n-non-linear slave systems with master system using non-adaptive and adaptive coupled observers," Energies, vol. 14, no. 11, p. 3190, 2021.

[20] P. N. Steinmetz, A. Roy, P. J. Fitzgerald, S. S. Hsiao, K. O. Johnson, and E. Niebur, "Attention modulates synchronized neuronal firing in primate somatosensory cortex," Nature, vol. 404, no. 6774, pp. 187-190, 2000.
[21] R. E. Mirollo and S. H. Strogatz, "Synchronization of pulsecoupled biological oscillators," SIAM Journal on Applied Mathematics, vol. 50, no. 6, pp. 1645-1662, 1990.

[22] R. L. Filali, M. Benrejeb, and P. Borne, "On observer-based secure communication design using discrete-time hyperchaotic systems," Communications in Nonlinear Science and Numerical Simulation, vol. 19, no. 5, pp. 1424-1432, 2014.

[23] M. Siddique, M. Hussain, M. Rehan, and M. M. Hussain, "Robust-adaptive synchronization of drive and response systems using coupled chaotic adaptive synchronous observers," IEEE Conference on Systems, Process and Control (ICSPC), pp. 24-29, 2017.

[24] A. Rodriguez-Angeles and H. Nijmeijer, "Mutual synchronization of robots via estimated state feedback: a cooperative approach," IEEE Transactions on Control Systems Technology, vol. 12 , no. 4 , pp. 542-554, 2004.

[25] L. Poinkam Meffo, P. Woafo, and S. Domngang, "Cluster states in a ring of four coupled semiconductor lasers," Communications in Nonlinear Science and Numerical Simulation, vol. 12, no. 6, pp. 942-952, 2007.

[26] L. Kuhnert, K. I. Agladze, and V. I. Krinsky, "Image processing using light-sensitive chemical waves," Nature, vol. 337, no. 6204, pp. 244-247, 1989.

[27] Y.-N. Li, L. Chen, Z.-S. Cai, and X.-z. Zhao, "Experimental study of chaos synchronization in the Belousov-Zhabotinsky chemical system," Chaos, Solitons \& Fractals, vol. 22, no. 4, pp. 767-771, 2004.

[28] Ö Morgül and E. Solak, "Observer based synchronization of chaotic systems," Physical Review. E, Statistical Physics, Plasmas, Fluids, and Related Interdisciplinary Topics, vol. 54, no. 5, pp. 4803-4811, 1996.

[29] J. F. Heagy, T. L. Carroll, and L. M. Pecora, "Synchronous chaos in coupled oscillator systems," Physical Review E, vol. 50, no. 3, pp. 1874-1885, 1994.

[30] S. Wen, Z. Zeng, and T. Huang, "Observer-based synchronization of memristive systems with multiple networked input and output delays," Nonlinear Dynamics, vol. 78, no. 1, pp. 541-554, 2014.

[31] M. Gerster, R. Berner, J. Sawicki et al., "FitzHugh-Nagumo oscillators on complex networks mimic epileptic-seizure-related synchronization phenomena," Chaos: An Interdisciplinary Journal of Nonlinear Science, vol. 30, no. 12, Article ID 123130, 2020.

[32] X. Sun, S. Wandelt, and A. Zhang, "On the degree of synchronization between air transport connectivity and COVID19 cases at worldwide level," Transport Policy, vol. 105, pp. 115-123, 2021. 